\title{
Two severe adverse events triggered by an anti-PD-1 immune checkpoint inhibitor in an advanced lung cancer patient: a case report and review of the literature
}

\author{
Zhiyao Bao $^{1,2}$, Xianwen Sun ${ }^{1,2}$, Wei Chen ${ }^{1,2}$, Wei Tang ${ }^{1,2}$, Beili Gao ${ }^{1,2}$, Yi Xiang ${ }^{1,2}$ \\ ${ }^{1}$ Department of Respiratory and Critical Care Medicine, Ruijin Hospital, Shanghai Jiao Tong University School of Medicine, Shanghai, China; \\ ${ }^{2}$ Institute of Respiratory Medicine, Shanghai Jiao Tong University School of Medicine, Shanghai, China \\ Correspondence to: Yi Xiang. Department of Respiratory and Critical Care Medicine, Ruijin Hospital, Shanghai, China; Institute of Respiratory \\ Medicine, Shanghai Jiao Tong University School of Medicine, Shanghai 200025, China. Email: xiangyiht@163.com.
}

\begin{abstract}
Anti-programmed death 1 (PD-1) immune checkpoint inhibitors have produced robust tumor responses in several solid tumors including lung cancer by enhancing the antitumor activity of the immune system. In general, the adverse events triggered by anti-PD-1/PD-L1 mAbs appear to be less severe when compared with traditional chemotherapy. However, a subgroup of patients will experience various autoimmune adverse events, such as skin, gastrointestinal, pulmonary, hepatic, renal, and endocrine events, among others. In previous studies, only one irAE was reported in a patient who received immunotherapy. However, in this report, we presented an advanced non-small cell lung cancer patient who was positive for PD-L1 in $20 \%$ of tumor cells and negative for actionable molecular markers such as KRAS, EGFR, ALK, MET, and ROS1 alterations. He received a PD-1 inhibitor combined with chemotherapy according to the guidelines of the Chinese Society of Clinical Oncology (CSCO) non-small cell lung cancer [2020] and experienced severe hepatitis and pneumonitis successively, which were recovered after the treatment of systemic glucocorticoids. This situation increased the difficulty of diagnosis and treatment of immune-related adverse events (irAEs). This case illustrates the potential toxicity caused by immunotherapy, and more attention should be paid to its prevention, treatment, and association with antitumor efficacy. Multidisciplinary discussions should be undertaken to improve patient prognosis.
\end{abstract}

Keywords: Immune-related adverse events (irAEs); advanced lung cancer; case report

Submitted Jun 17, 2021. Accepted for publication Aug 23, 2021.

doi: $10.21037 /$ atm-21-4167

View this article at: https://dx.doi.org/10.21037/atm-21-4167

\section{Introduction}

Immunotherapy with monoclonal antibodies (mAbs) can block programmed death 1 (PD-1)-dependent negative regulation of the immune response. Hence, it has been a major success story in the treatment of several cancer types including lung cancer. In spite of this achievement, in a subgroup of patients, anti-PD-1 therapy has been associated with several immune-related adverse events (irAEs) such as rash, pruritus, thyroiditis, diarrhea, nephritis, hepatitis, and pneumonitis. IrAEs resulting from immune checkpoint inhibitor (ICI) therapy are common, and are often not severe enough to lead to treatment interruptions (1).
However, severe irAEs can be life threatening in some cases. In this manuscript, we report a case of immunotherapyrelated liver dysfunction and immunotherapy-related pneumonia following the first cycle treatment of camrelizumab combined with chemotherapy (Figure 1). We present the following article in accordance with the CARE reporting checklist (available at https://dx.doi.org/10.21037/ atm-21-4167).

\section{Case presentation}

In May 2020, a 62-year-old man was diagnosed with stage 


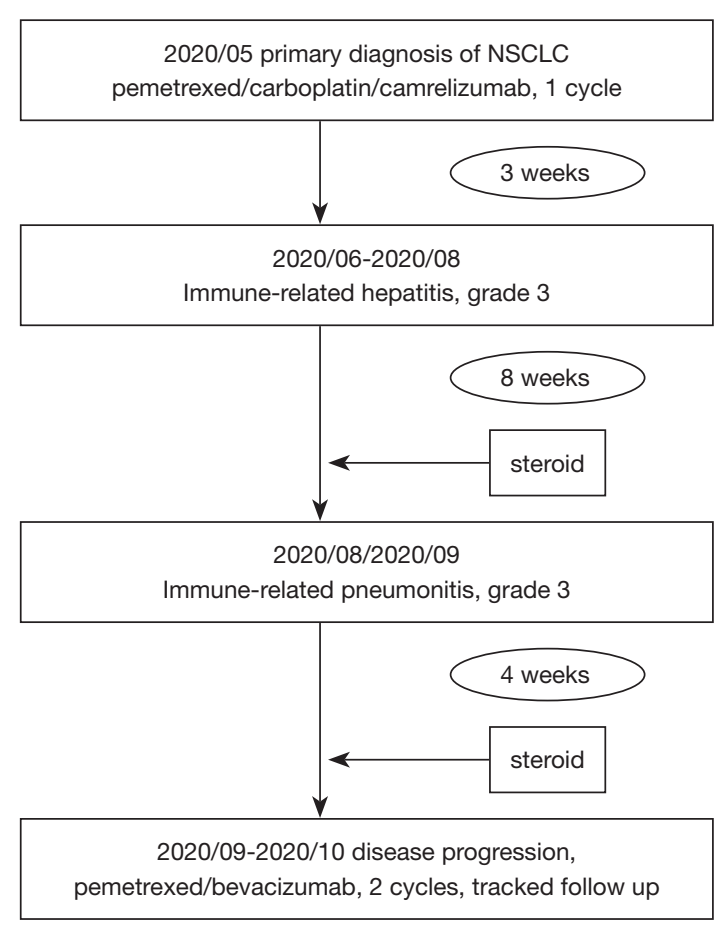

Figure 1 The axis of anti-tumor treatment and intervention on immune-related adverse events (irAEs).

IV left lung adenocarcinoma (Figure $2 A$ ) which was negative for KRAS, EGFR, ALK, MET, and ROS1 alterations and positive for PD-L1 in $20 \%$ of tumor cells. He was an HBV carrier and had a history of percutaneous transluminal coronary angioplasty with stent implantation 4 years ago. It's reported that patients with high expression of PD-L1 (positive in $>50 \%$ of tumor cells) could be treated with mono immunotherapy; and patients with $1-49 \%$ positive of PD-L1 in tumor cells, immunotherapy combined with chemotherapy were recommended. Since the patient had an excellent performance status and no history of autoimmune disorders, he received first-line chemotherapy with a combination of ICIs according to the guidelines of the Chinese Society of Clinical Oncology (CSCO) non-small cell lung cancer [2020]. The medicines were as follows: pemetrexed $\left(500 \mathrm{mg} / \mathrm{m}^{2} \mathrm{BSA}\right)$, carboplatin $(\mathrm{AUC}=5)$, and camrelizumab $(200 \mathrm{mg})$ on day 1.

After 3 weeks, the patient was admitted to our department for the second cycle of treatment. Routine biochemical laboratory blood tests showed abnormal liver function, as alanine aminotransferase (ALT) was $280 \mathrm{U} / \mathrm{L}$ and aspartate aminotransferase (AST) was $268 \mathrm{U} / \mathrm{L}$. However, the patient was asymptomatic. Meanwhile, the patient received a chest CT scan which showed that the primary lesion was stable (Figure 2B). Treatment of advanced lung cancer was interrupted, and the patient-initiated therapy for abnormal liver function with reduced glutathione, diammonium glycyrrhizinate, and polyene phosphatidylcholine. Furthermore, his liver function became worse with the appearance of jaundice (Table 1), and the patient felt fatigue and lack of appetite. Ursodeoxycholic acid combined with ademetionine was added to improve liver function. Meanwhile, a series of tests were performed to determine the cause of liver dysfunction. Since the patient was an $\mathrm{HBV}$ carrier, hepatitis A, B, C, and E tests were performed to exclude active hepatitis. HBV-DNA was normal and antibodies for the other hepatitis viruses were negative. Cytomegalovirus (CMV) and human immunodeficiency virus (HIV) were negative. Extended liver autoimmune antibodies were also all negative. An MR scan of the liver showed only 2 small liver metastases and no biliary dilatation. After the patient received a liver puncture (Figure 3), a multidisciplinary discussion was conducted, including a liver specialist, pathologist, and respiratory specialist. Immune-related hepatitis was considered, and as per the Chinese experts consensus on immune checkpoint inhibitors for non-small cell lung cancer (2019 version) (2), $60 \mathrm{mg}$ $(1.5 \mathrm{mg} / \mathrm{kg} / \mathrm{d})$ of intravenous methylprednisolone twice a day was given. After 7 days of therapy with the glucocorticoid and other hepatoprotective drugs, although the patient's serum transaminase and bilirubin levels remained stable, he felt better. The patient was then transferred to the department of infectious disease, and with the support of liver pathological results, the patient continuously received intravenous methylprednisolone. After a total of 14 days of therapy with $120 \mathrm{mg} / \mathrm{d}$ methylprednisolone, the patient's liver function improved, and the dose of methylprednisolone was decreased to $80 \mathrm{mg} / \mathrm{d}$. With the continuous improvement of the patient's serum transaminase and bilirubin levels, methylprednisolone was gradually reduced to oral administration and suspended. The total duration of methylprednisolone therapy was approximately 6 weeks. Before discharge, the patient received another chest CT scan, however the result was unheeded

At 10 days after methylprednisolone withdrawal, the patient was followed up by phone. He felt chest tightness and shortness of breath, and showed progressive aggravation. He was admitted to hospital again immediately, his oxygen saturation $\left(\mathrm{SpO}_{2}\right)$ was $52 \%$ at room air, but improved to $92 \%$ on high-flow oxygen with $90 \%$ oxygen concentration. On physical examination, his respiration was 

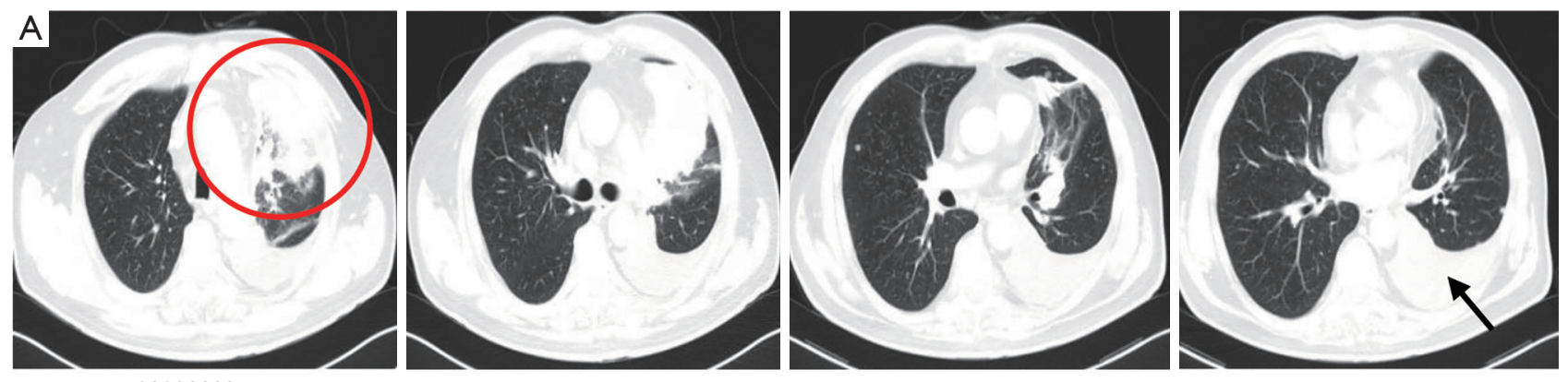

20200603
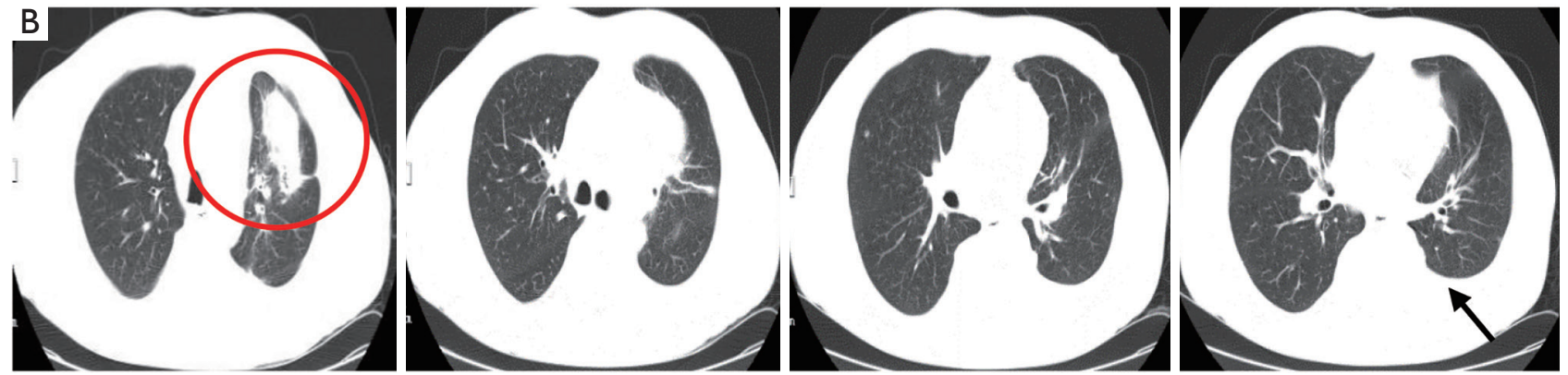

20200625

Figure 2 Dynamic changes of patients' CT scan. (A) Base line, a CT scan showed primary lesion in the upper lobe of left lung near the hilum, with the largest section about $7.8 \mathrm{~cm} \times 7.5 \mathrm{~cm}$, left pleural effusion. (B) After receiving 1 cycle treatment of pemetrexed/carboplatin/ camrelizumab, a CT scan showed a stable situation of lung lesions. Circle indicates primary lesion, black arrow indicates pleural effusion.

Table 1 The trend of liver function

\begin{tabular}{|c|c|c|c|c|c|}
\hline Live function & \multicolumn{5}{|c|}{ Date } \\
\hline Alanine aminotransferase (U/L) [10-64] & 39 & 280 & 279 & 200 & 159 \\
\hline Aspartate aminotransferase (U/L) [8-40] & 39 & 268 & 214 & 214 & 121 \\
\hline Alkaline phosphatase (U/L) [38-126] & 158 & 259 & 295 & 295 & 407 \\
\hline Direct bilirubin $(\mu \mathrm{mol} / \mathrm{L})(0-6.8)$ & 3.4 & 3.9 & 24.1 & 46.7 & 66.4 \\
\hline
\end{tabular}

labored, bilateral wheezes and crackles were auscultated, and the remainder of his examination was unremarkable including normal temperature. Arterial blood gas (ABG) demonstrated that the oxygenation index was less than $200 \mathrm{mmHg}$. A chest X-ray (CXR) showed bilateral interstitial opacities consistent with either pneumonia or pneumonitis (Figure 4A). At first, he received anti-infective therapy (meropenem 1.0 quaque 8 hours + voriconazole $200 \mathrm{mg} / \mathrm{d}+$ compound sulfamethoxazole 3 OC three times daily) and $60 \mathrm{mg}(1.5 \mathrm{mg} / \mathrm{kg} / \mathrm{d})$ of intravenous methylprednisolone twice a day. Meanwhile, laboratories tests were performed to identify whether he had pneumonia or pneumonitis. White cell count, plasma procalcitonin level, $\beta-1,3$-glucan test, galactomannan test, and latex agglutination test were normal. Sputum microbial testing, including staining and culture for bacteria, fungi, and mycobacteria, were negative. Cytological analysis for malignant cells was also negative. The patient was intolerant to bronchoscopy, hence his sputum was sent for metagenomics next generation sequencing. Sputum testing returned negative for Leginella, Histoplasma capsulatum, Aspergillus, Pneumocystic jiroveci, and respiratory 

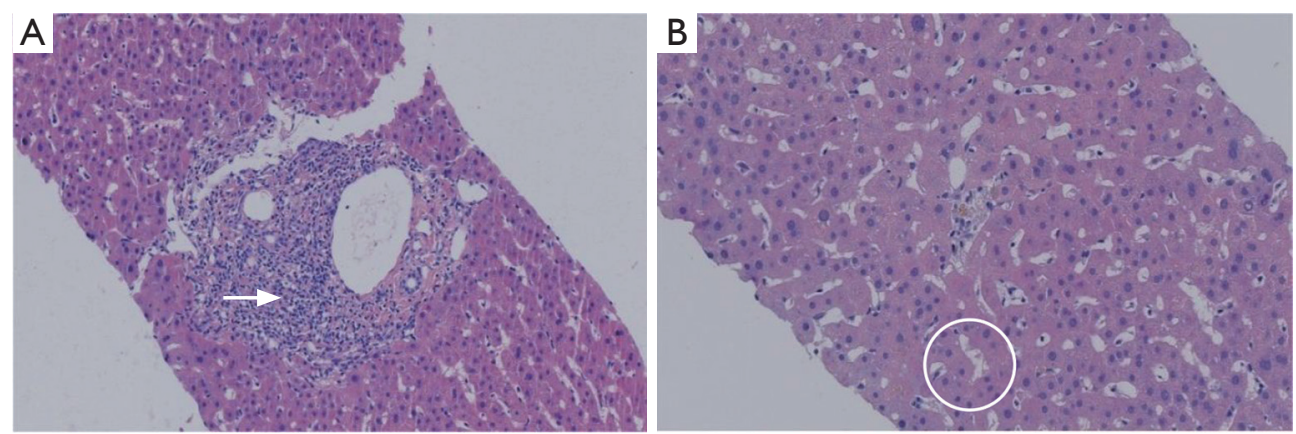

Figure 3 Liver histology (haematoxylin \& eosin staining). The biopsy showed focal necrosis and eosinophilic bodies, a small number of hepatic cells express fat denaturation, a small amount of hepatocyte cholestasis and feathery degeneration, the arrangement of lymphocytes in hepatic sinusoid and mild inflammation in portal area. (A) White arrow indicates lymphocytes aggregation $(\times 40)$; (B) circle indicates hepatocyte necrosis and cholestasis $(\times 100)$.

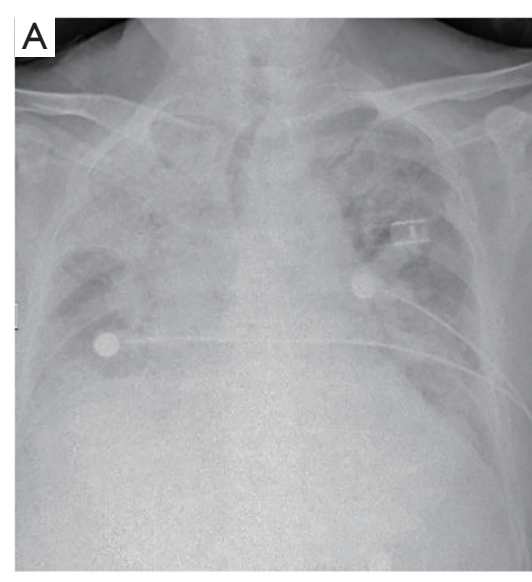

20200824

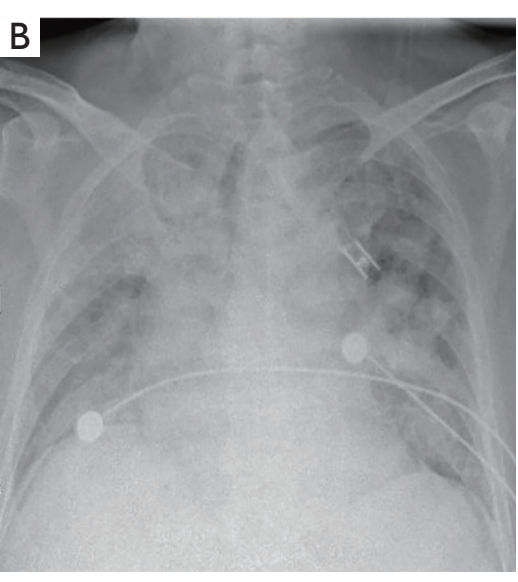

20200826

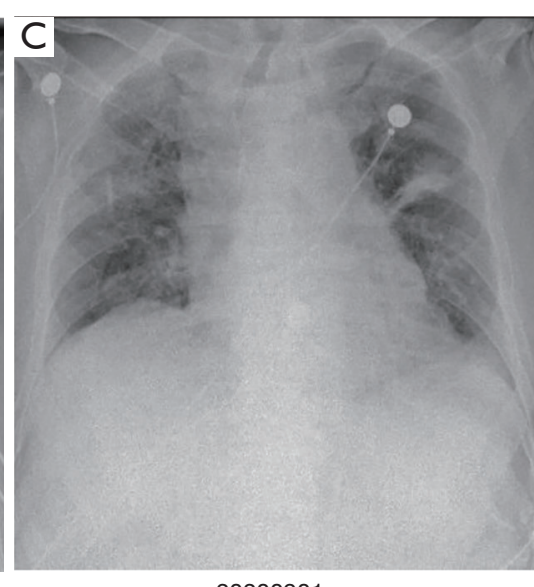

20200901

Figure 4 Bedside chest X-ray showed immune-related pneumonitis. (A) Bedside chest X-ray showed patchy ground-grass opacities in the bilateral lungs; (B) after treatment with corticosteroid for 2 days, the patient's symptom without deterioration, with partial improvement of the ground-glass opacities; (C) after treatment with corticosteroid for 7 days, the majority absorption of ground-glass opacities, with significantly symptom improved of the patient's.

viruses (including influenza virus, adenovirus, and CMV, among others). We also noticed that his chest CT scan, which was taken 10 days before this hospital admission, showed new ground-grass opacities in the lung bilaterally. Based on his clinical presentations and laboratory tests, the patient was diagnosed with grade 3 ICI-induced pneumonitis. Anti-infective drugs were gradually discontinued while intravenous methylprednisolone $(1.5 \mathrm{mg} / \mathrm{kg} / \mathrm{d})$ was maintained. At 7 days later, his dyspnoea improved, his $\mathrm{SpO}_{2}$ had increased to $94 \%$ at room air, and his CXRs showed gradual improvement of transparency (Figure 4B,4C). He received another 7 days of intravenous methylprednisolone $(1 \mathrm{mg} / \mathrm{kg} / \mathrm{d})$, followed by conversion to $1 \mathrm{mg} / \mathrm{kg} / \mathrm{d}$ peroral methylprednisolone. After a CT scan (Figure $5 A, 5 B$ ) showed some absorption of bilateral opacities, he was discharged on a dose of $1 \mathrm{mg} / \mathrm{kg} / \mathrm{d}$ peroralmethylprednisolone.

At 2 weeks later, he was readmitted to hospital for worsening dyspnoea and a $\mathrm{SpO}_{2}$ of $88 \%$ at room air. Left massive hydrothorax was found (Figure 5C), and disease progression was confirmed by positive cytological analysis of pleural effusion. It was 105 days later after the patient received first line chemotherapy combined with immunotherapy. He received pemetrexed $\left(500 \mathrm{mg} / \mathrm{m}^{2}\right.$ BSA $)$ for another 2 chemotherapy cycles, and remained stable. There were no further ICI-related toxicities. 

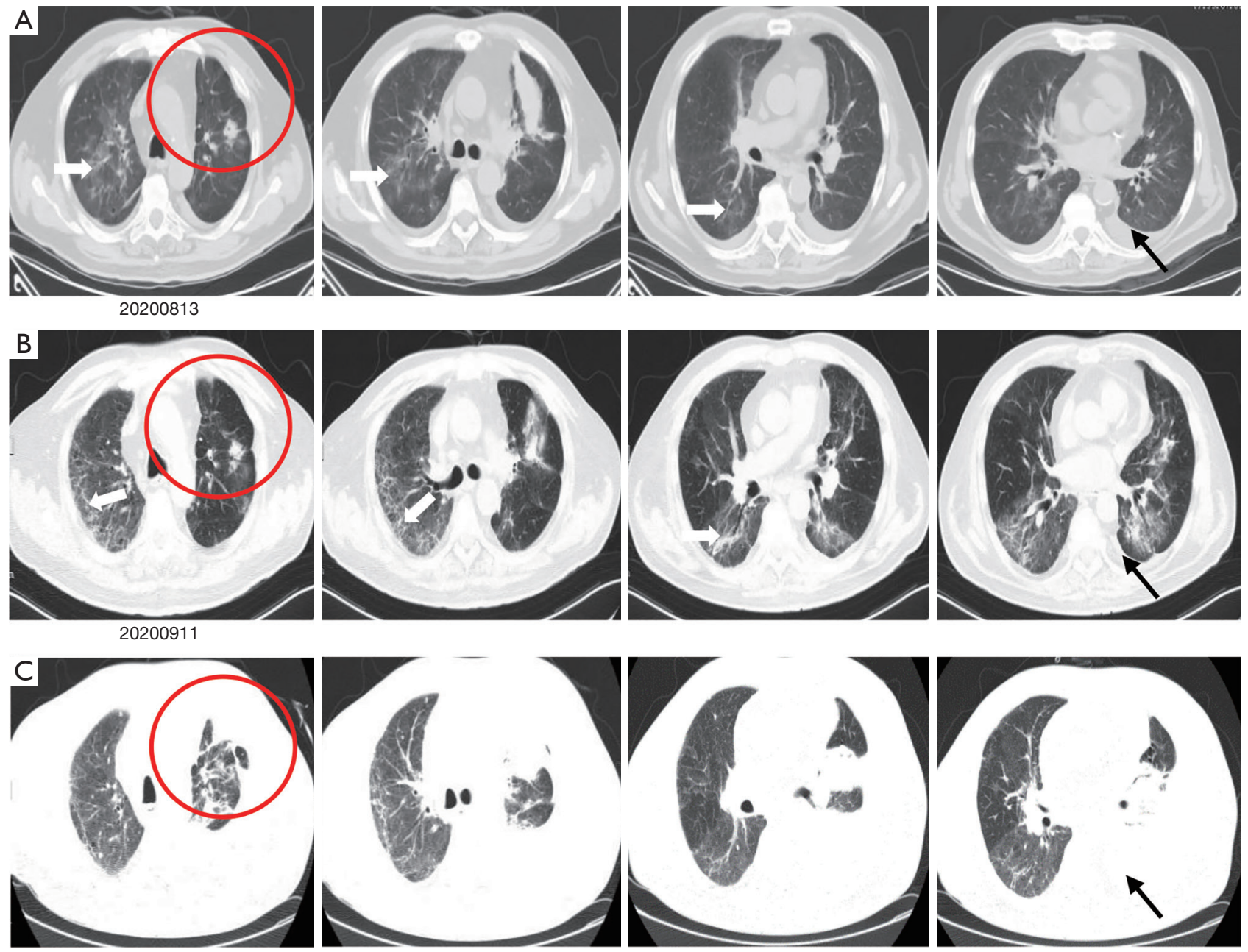

20200925

Figure 5 Follow up of patients' CT scan. (A) In the end of receiving steroid treatment of immune-related hepatitis, new patchy opacity developed mainly in the right lung; (B) during receiving steroid treatment of immune-related pneumonitis, pneumonitis was partially resolved nearly 1month, with a significantly clinical improvement; (C) disease progression, left pleural effusion was increased. Circle indicates primary lesion, black arrow indicates pleural effusion, white arrow indicates patchy ground-grass opacities.

All procedures performed in studies involving human participants were in accordance with the ethical standards of the institutional and/or national research committee(s) and with the Helsinki Declaration (as revised in 2013). Written informed consent was obtained from the patient for publication of this case report and accompanying images. A copy of the written consent is available for review by the editorial office of this journal.

\section{Discussion}

The capacity to exploit the host immune system to treat cancer is known as immunotherapy, which has demonstrated efficacy in the treatment of different cancer types. In lung cancer therapy, the evaluation of immunotherapy efficacy is RECIST v1.1, which is the same as chemotherapy. Among the different treatments that this therapy encompasses, PD-1/PD-L1 inhibitors can strengthen the function of effector $\mathrm{T}$ cells and increase antitumor reactions. However, if there is an excessive response, it can damage normal tissue, which results in irAEs in a manner similar to autoimmune disorders such as rash, pruritus, thyroiditis, diarrhea, nephritis, hepatitis, and pneumonitis. (3). The irAEs could happen as soon as the immunotherapy started in no matter what kind of cancer. It has become a new challenge for us to select the dominant population, plan the therapy, evaluate 
the efficacy, and identify and deal with the irAEs.

Immune-related hepatitis is characterized by increased liver function test results, mainly AST, ALT, and glutamyl transpeptidase (GGT) levels, but rarely bilirubin, without symptoms. Anti-PD-1/PD-L1 mAbs are associated with elevated AST and ALT levels in $5 \%$ of patients or less, with grade $3 / 4$ events occurring in $1-2 \%$ of patients, which are less than those induced by anti-CTLA4 mAbs (4). Reports have shown that the median time of the appearance of hepatic dysfunction from the start of PD-1 inhibitor therapy is about 41 days (range, 21-120 days) (5). A diagnosis of immune-related hepatitis requires ruling out active viral hepatitis, progression of primary liver tumor or liver metastasis, liver injury caused by other drugs, liver injury caused by other diseases (such as fatty liver, alcoholic liver, etc.), and autoimmune hepatitis, among other conditions. In our case, the patient showed increased ALT and AST levels after the first cycle of chemotherapy combined with camrelizumab. With conventional treatment, liver function worsened followed by an increase in serum bilirubin. Active viral hepatitis, autoimmune hepatitis, and liver metastasisinduced liver injury were ruled out by the detection of antibodies and examination of imaging (abdominal CT and liver MRI). The patient showed a rs72631567 mutation on chromosome 2, which is associated with drug-induced liver injury (DILI) (6). There are few reports on liver injury caused by carboplatin, and the incidence of liver injury caused by pemetrexed is between $1-10 \%$, which can recover quickly by the application of a liver protectant. Furthermore, a liver puncture was performed, and the histological results demonstrated lobular hepatitis with lymphohistiocytic inflammation and hepatocyte injury. After multidisciplinary discussion, immune-related hepatitis was diagnosed and systemic glucocorticoids were used according to the consensus. Liver function improved gradually and the dosage of glucocorticoids was adjusted. Approximately 6 weeks later, the patient recovered from immune-related hepatitis.

Immune-related pneumonitis associated with anti-PD-1 or anti-PD-L1 monotherapy is relatively rare, and the incidence is roughly $3 \%$, but it is potentially life-threatening (7). It is usually shown as diffuse, centrilobular nodules and patchy ground-glass attenuation with areas of air-trapping on CXR or thoracic CT, which is non-infectious lung inflammation (8). Pneumonitis manifests as tachypnoea, dyspnea, cyanosis, fatigue, unproductive cough, tachycardia, seldom fever, and chills. A diagnosis of pneumonitis requires ruling out all types of infectious pulmonary inflammation, mainly virus-related or triggered by atypical pneumonia, cancer progression, and radiation-induced pneumonitis. Several events may increase the risk of developing immunerelated pneumonitis, such as previous use of cytotoxic drugs (taxanes, gemcitabine) and tyrosine kinase inhibitors, preexisting pulmonary damage from inflammation, radiation, idiopathic pulmonary disease, as well as increased tumor burden (9). Cases are typically mild, but $1 \%$ of patients will develop severe (grade 3 or grade 4) pneumonitis. In this case, our patient gradually developed dyspnea and serious interstitial lung disease during the treatment of immunerelated hepatitis. However, he showed neither signs of tumor progression nor signs of infection at the time of admission, and he received glucocorticoids again with the protection of antibiotics. About 2 weeks later, the patient's oxygenation gradually improved and interstitial pulmonary changes were absorbed, hence, no other immunosuppressive agents were used.

\section{Conclusions}

Combined therapy with PD-1/PD-L1 inhibitors has been the first-line treatment for PD-L1 positive advanced nonsmall cell lung cancer. Early detection and treatment of irAEs pose a new challenge to clinicians. They can cause collateral damage to multiple systems including the endocrine system, skin, gastrointestinal system, hepatic system, pulmonary system, and mucocutaneous system, among others, which may occur simultaneously or successively. Diagnostic routine procedures should be performed, patients should be informed and trained to report symptoms as early as possible to their treating physician, and multidisciplinary discussions should be arranged to inform treatment. Due to the potentially late onset of irAEs, patients should be monitored at regular intervals, combining clinical symptoms, such as fatigue, rash, diarrhea, and laboratory inspection, such as liver function, kidney function, endocrine function, CXR/CT, etc. should be done to monitor the adverse reactions in the follow-up course.

\section{Acknowledgments}

Funding: This project has been funded in part by grant No. 81600004 from the National Nature Science Foundation of China and Key Laboratory of Emergency Prevention, Diagnosis and Treatment of Respiratory Infectious Diseases, Shanghai (No. 20dz2261100). 


\section{Footnote}

Reporting Checklist: The authors have completed the CARE reporting checklist. Available at https://dx.doi. org/10.21037/atm-21-4167

Conflicts of Interest: All authors have completed the ICMJE uniform disclosure form (available at https://dx.doi. org/10.21037/atm-21-4167). The authors have no conflicts of interest to declare.

Ethical Statement: The authors are accountable for all aspects of the work in ensuring that questions related to the accuracy or integrity of any part of the work are appropriately investigated and resolved. All procedures performed in studies involving human participants were in accordance with the ethical standards of the institutional and/or national research committee(s) and with the Helsinki Declaration (as revised in 2013). Written informed consent was obtained from the patient for publication of this case report and accompanying images. A copy of the written consent is available for review by the editorial office of this journal.

Open Access Statement: This is an Open Access article distributed in accordance with the Creative Commons Attribution-NonCommercial-NoDerivs 4.0 International License (CC BY-NC-ND 4.0), which permits the noncommercial replication and distribution of the article with the strict proviso that no changes or edits are made and the original work is properly cited (including links to both the formal publication through the relevant DOI and the license). See: https://creativecommons.org/licenses/by-nc-nd/4.0/.

\section{References}

1. Kottschade LA. Incidence and Management of ImmuneRelated Adverse Events in Patients Undergoing Treatment with Immune Checkpoint Inhibitors. Curr Oncol Rep

Cite this article as: Bao Z, Sun X, Chen W, Tang W, Gao B, Xiang Y. Two severe adverse events triggered by an anti-PD-1 immune checkpoint inhibitor in an advanced lung cancer patient: a case report and review of the literature. Ann Transl Med 2021;9(16):1358. doi: 10.21037/atm-21-4167
2018;20:24.

2. Zhou C, Wang J, Bu H, et al. Chinese Experts Consensus on Immune Checkpoint Inhibitors for Non-small Cell Lung Cancer (2019 version). Zhongguo Fei Ai Za Zhi 2020;23:65-76. Erratum in: Zhongguo Fei Ai Za Zhi 2020;23:210.

3. Stucci S, Palmirotta R, Passarelli A, et al. Immunerelated adverse events during anticancer immunotherapy: Pathogenesis and management. Oncol Lett 2017;14:5671-80.

4. Naidoo J, Page DB, Li BT, et al. Toxicities of the antiPD-1 and anti-PD-L1 immune checkpoint antibodies. Ann Oncol 2015;26:2375-91. Erratum in: Ann Oncol 2016;27:1362.

5. Mathew Thomas V, Bindal P, Ann Alexander S, et al. Nivolumab-induced hepatitis: A rare side effect of an immune check point inhibitor. J Oncol Pharm Pract 2020;26:459-61.

6. Nicoletti P, Aithal GP, Bjornsson ES, et al. Association of Liver Injury From Specific Drugs, or Groups of Drugs, With Polymorphisms in HLA and Other Genes in a Genome-Wide Association Study. Gastroenterology 2017;152:1078-89.

7. Naidoo J, Wang X, Woo KM, et al. Pneumonitis in Patients Treated With Anti-Programmed Death-1/ Programmed Death Ligand 1 Therapy. J Clin Oncol 2017;35:709-17. Erratum in: J Clin Oncol 2017;35:2590.

8. Eigentler TK, Hassel JC, Berking C, et al. Diagnosis, monitoring and management of immune-related adverse drug reactions of anti-PD-1 antibody therapy. Cancer Treat Rev 2016;45:7-18.

9. Nishino M, Chambers ES, Chong CR, et al. Anti-PD-1 Inhibitor-Related Pneumonitis in Non-Small Cell Lung Cancer. Cancer Immunol Res 2016;4:289-93.

(English Language Editor: C. Betlzar) 